oder in epikardialen Regionen des linken Ventrikels.,Etwa 20\% der LOVT-Tachykardien haben ihren Ursprung in der Aortenwurzel", so Reithmann. Am häufigsten ist der linkskoronare Sinus betroffen. Ähnlich wie bei RVOTTachykardien muss auch bei LOVT-Tachykardien immer eine strukturelle Schädigung diskutiert und, soweit möglich, ausgeschlossen werden. „Kernspintomografisch findet sich nicht selten eine Fibrosierung des superioren Ventrikel-Septums, sodass es sich eigentlich nicht um eine idiopathische Rhythmusstörung handelt", so Reithmann.

Im Unterschied zu den anderen idiopathischen ventrikulären Tachykardien sollte bei einer epikardialen linksventrikulären Ausflusstrakt-Tachykardie, die von dem linksventrikukären Summit ausgeht, immer die Ablation über eine Perikardpunktion durchgeführt werden.

Eine besondere Form der benignen ventrikulären Tachykardie ist die idiopathische linksventrikuläre Reentry-Tachykardie. Elektrophysiologisch entsteht die Rhythmusstörung durch Mikro-Reentrys. Solche Patienten sollten vorrangig immer mit einer Katheterablation behandelt werden. Die Implantation eines automatischen Defibrillators ohne Ablation ist nicht sinnvoll, da bei solchen Patienten ansonsten gehäuft inadäquate Schocks abgegeben würden. „Bei dieser Rhythmusstörung handelt es sich um eine faszikuläre Tachykardie, die auch dann erfolgreich abladiert werden kann, wenn sie nicht induzierbar ist", so Reithmann. Differenzialdiagnostisch müsse bei einer solchen linksposterioren Faszikeltachykardie aber auch immer eine Tachykardie ausgehend vom posterioren Papillarmuskel diskutiert werden. Auch finde sich bei solchen Patienten nicht selten kernspintomografisch eine minimale strukturelle Herzerkrankung im Sinne einer Fibrosierung.

\section{VT-Ablation bei struktureller Herzerkrankung}

Patienten mit ventrikulären Tachykardien bei struktureller Herzerkrankung haben ein hohes Risiko für den akuten Herztod. Deshalb erfordern sie meist die Implantation eines ICD. Doch um unnötige Schockabgaben zu verhindern, sollte bei solchen ventrikulären Tachykardien zusätzlich immer eine Substrat-basierte, elektrophysiologisch geführte Ablation erfolgen. Dabei wird zunächst ein dreidimensionales strukturelles Mapping durchgeführt und anschließend wird versucht, mit Stimulationsmanövern die Rhythmusstörung zu induzieren. Probleme können bei Patienten mit multipler VT-Morphologie oder bei instabilen ventrikulären Tachykardien auftreten.

„Die Katherablation einer ventrikulären Tachykardie bei struktureller Herzerkrankung ist heute ein etabliertes und sehr gut wirksames Therapieverfahren mit einer Erfolgsrate von ca. $80 \%$ ", so Reithmann. Bei Patienten mit einer ischämischen Kardiomyopathie kann in 65\% der Fälle nach diesem Eingriff keine monomorphe ventrikuläre Tachykardie mehr ausgelöst werden und bei $28 \%$ der Patienten ist der Therapieerfolg unbestimmt, d. h. es finden sich nach dem Eingriff monomorphe ventrikuläre Tachykardien anderer Morphologie und Zykluslänge. Nur bei 7\% der Patienten sind die ventrikulären Tachykardien weiterhin auslösbar im Sinne eines Therapieversagens. Bei Patienten mit nicht ischämischer Kardiomyopathie sind die Erfolgsraten etwas schlechter mit einer kompletten Erfolgsrate von nur 51\% und einem Therapieversagen bei $21 \%$ der Patienten.

Dr. med. Peter Stiefelhagen I

|[Quelle: 2. Rhythmologie-Update-Seminar, 17.11.2011 in Wiesbaden

\title{
Koronarverschluss nach Infarkt
}

\section{Subakute Rekanalisation langfristig ohne Nutzen?}

\author{
Es bleibt dabei: Infarktpatienten müs- \\ sen schnellstmöglich ins Spital, um das \\ Infarktgefäß zu rekanalisieren. Eine ver- \\ zögerte Wiedereröffnung verschlos- \\ sener Koronarien bringt auch langfristig \\ keinerlei Nutzen.
}

Hat der Patient eventuell auch noch einen Vorteil, wenn verschlossene Koronararterien subakut ab dem zweiten Tag nach dem Infarkt perkutan wiedereröffnet werden?

Dieser Frage ging der Occluded Artery Trial bei 2201 stabilen Infarktpatienten nach und fand nach einem Follow-up von im Schnitt 2,9 Jahren heraus: Der Eingriff redu- ziert weder die Mortalität noch das Risiko für Reinfarkte oder jenes für die Entwicklung einer Herzinsuffizienz.

Auch langfristig zeigt sich leider kein Vorteil für den Eingriff, so das Ergebnis der Nachbeobachtung über im Schnitt sechs Jahre, die jetzt in Circulation online publiziert wurde: Die Rate der klinischen Komplikationen waren nach PTCA und alleiniger medikamentöser Therapie exakt gleich.

Nur 14,7 Prozent der konservativ behandelten Patienten mussten sich binnen sechs Jahren einem Kathetereingriff unterziehen, aber auch 11,1 Prozent der schon zu Studienbeginn mit PTCA behandelten Patienten.
Nur wenige Patienten mit Stenokardien Auch die Beschwerdesymptomatik rechtfertigt den Eingriff nicht: Nur sehr wenige Patienten litten während des Follow-ups noch unter pektanginösen Beschwerden.

Zwar waren die interventionell behandelten Patienten insgesamt etwas weniger symptomatisch. Die Differenz symptomatischer Patienten zwischen den Behandlungsgruppen lag aber stets unter 4 Prozent.

Dr. med. Dirk Einecke II

I| Quelle: Hochman J.S. et al. Circulation 2011; 124:2320-8. DOI: 10.1161/CIRCULATI-

ONAHA.111.041749 